\title{
Integrative representations and analyses of vaccine-induced intended protective immunity and unintended adverse events using ontology-based and theory-guided approaches
}

\author{
Yongqun He*, Edison Ong, and Jiangan Xie \\ University of Michigan Medical School, USA
}

While effective preventive vaccines induce intended protective immunity, they also induce unintended adverse events (AEs). Generally speaking, compared to killed, inactivated vaccines and protein vaccines, live attenuated vaccines induce more protective immune responses. However, live attenuated vaccines are also associated with more AEs and even more serious AEs. For example, while live attenuated smallpox vaccines were critical to the eradication of smallpox, approximately 20 $30 \%$ of smallpox vaccine recipients also experienced with various AEs that range in prevalence and severity [1]. Inter-individual variations in cytokine and $\mathrm{AE}$ response after smallpox vaccinations are in part due to genetic variation. For another example, the attenuated oral poliovirus vaccine (OPV) efficiently induces intestinal immunity and durable humoral immunity. However, OPV has the disadvantage of genetic instability, contributing to rare and sporadic cases of vaccineassociated paralytic poliomyelitis and the emergence of genetically divergent vaccine-derived polioviruses [2]. These AEs are worsened in patients with primary immunodeficiencies. These results suggest that the intended protective immune responses and unintended adverse events are correlated and deserve being studied simultaneously.

A biomedical ontology is a human- and computer-interpretable set of terms and relations that represent entities in a specific biomedical domain and how they relate to each other. Ontologies have emerged to be critical to biomedical data and knowledge representation, exchange, integration, as well as inferring new knowledge. We have initiated and led the development of three vaccine-focused ontologies:

(i) The Vaccine Ontology (VO) is developed to ontologically represent licensed vaccines or experimentally verified vaccine candidates, vaccine components, and host immune responses to vaccines $[3,4]$. The VO has also represented key vaccine information manually curated from the literature and stored in the VIOLIN vaccine database $[5,6]$. The hierarchies and logical relations of thousands of terms in VO support computer-assisted automated inferencing. VO has been applied to support various vaccine data integration [7-11] and literature mining $[4,12,13]$.

(ii) The Ontology of Vaccine Adverse Events (OVAE) is an ontology of the AEs known to be associated with the administration of licensed vaccines [14]. By extending VO and the Ontology of Adverse Events (OAE) [15], OVAE effectively imports the information of vaccines and adverse events. With these imported terms, OVAE is able to semantically define vaccine-specific adverse events. Based on the official FDA licensed vaccine package insert documents, age-specific $\mathrm{AE}$ occurrence rates in different populations are also represented for specific vaccine-specific adverse events (e.g., influenza vaccine Afluria- associated pain AE). Currently, OVAE includes all over 1,300 AEs associated with 63 US-licensed human vaccines [14]. OVAE can be easily used to query for important vaccine safety questions such as the most common VAEs observed in humans and the vaccines that have most AEs [14].

(iii) The Vaccination Informed Consent Ontology (VICO) is an ontology targeted to integrate different forms of vaccination informed consent forms with the goal of supporting integrative informed consent form query and result analysis [16]. Although signing a vaccination informed consent is not required at the federal level in the USA and Canada, many pharmacies (e.g., Costco and Walgreens) and state level regulations (e.g., Canada Manitoba government Public Health division) require informed consent before specific vaccinations. The vaccination informed consent forms from these different sources are different, making it difficult to integrate these forms and patients' records for advanced studies. VICO provides an ontological solution to such an issue [16]. In addition, by importing vaccine information from $\mathrm{VO}$ to the VICO, it is possible to make advanced inference. For example, based on vaccine contraindication information (from $\mathrm{VO}$ ) and a user's answer to an allergic question(s) in a vaccination informed consent form, we may infer a possible disqualification of the user for the vaccination due to a contraindication-related safety concern [15].

As described above, VO, OVAE, and VICO focus on the representation and analysis of vaccine formulations, vaccine safety, and vaccination informed consents, respectively. One missing domain of representation is the fundamental causal molecular interactions that eventually result in clinical phenotypes, which can be intended immune protection and unintended adverse events. How to better study the transition from basic molecular mechanisms to clinical phenotypes has remained a huge challenge.

In a recent publication [17], an ontological semantic framework is reported that links biological mechanisms to phenotypes of AEs by combining OAE with MedDRA in clinical FAERS drug case report data analysis. The Medical Dictionary for Regulatory Activities (MedDRA) is the default dictionary for AE reporting in the FDA AE Reporting System (FAERS). In this study, the AEs associated with five Tyrosine Kinase Inhibitors (TKIs) and monoclonal antibodies (mAbs) targeting tyrosine kinases, which induce impaired ventricular function (non-

Correspondence to: Yongqun He, University of Michigan Medical School, Ann Arbor, MI 48109, USA, Tel: 734-615-8231, Email: yongqunh@med.umich.edu

Received: May 04, 2016; Accepted: May 18, 2016; Published: May 23, 2016 
He Y (2016) Integrative representations and analyses of vaccine-induced intended protective immunity and unintended adverse events using ontology-based and theory-guided approaches

QT) cardiotoxicity, were investigated. Statistical analysis of FAERS data identified 1,053 distinct MedDRA terms significantly associated with TKIs/mAbs. These MedDRA terms were mapped to OAE terms that also link to other existing ontologies including the Gene Ontology (GO) [18]. The relations between TKIs/mAbs, cardiotoxicity AEs, and underlying gene products were generated. Such a study demonstrates that the combination of OAE and MedDRA provides a semantic framework to link clinical phenotypes of adverse drug events to biological mechanisms.

Theories provide guidance and hypotheses to practical studies. For understanding vaccine responses, two theories have been recently proposed. First, Dr. Gregory A Poland proposed an "Immune Response Gene Network Theory" $[19,20]$ which states that the responses to a vaccine are the cumulative results of interactions driven by a host of genes and the interactions among these genes in a choreographed fashion. Important immune response genes, gene polymorphisms, epigenetic modifications, and gene-gene interactions may all change the outcomes of host immune responses to a vaccine [19]. This theory has been used to guide the studies of the associations between immune response gene polymorphisms and various vaccine-induced antibody and cell-mediated immune responses [19] and the development of vaccinomics [21] and adversomics strategies [22].

More recently, Dr. Yongqun He proposed "the OneNet Theory of Life" $[23,24]$. The OneNet theory treats the whole process of a life of an individual organism as one single complex and dynamic network (abbreviated as OneNet). The OneNet has four characteristics that cover the OneNet Blueprint, OneNet Start, OneNet Dynamics, and OneNet Effectiveness [24]. Compared to the Immune Response Gene Network Theory, the OneNet theory is more focused on the exploration of the root cause of an organism's phenotypes such as vaccine-induced immune responses and AEs. The OneNet theory targets the systematic representation and analysis of the life of one organism (e.g., a human being), with a special focus on the dynamic interactions among genotype, environments, and phenotypes along the life process. Based on the OneNet theory, it is hypothesized that one human uses one single genotype-rooted mechanism (i.e., OneNet blueprint) to respond to different vaccinations [24]. This hypothesis appears contradictory against obvious experimental observations that human uses different mechanisms against different vaccines. However, experimentally identified mechanisms are hypothesized to be different manifestations of the same OneNet blueprint mechanism under specific conditions [24]. The introduction of the two concepts, OneNet blueprint and OneNet manifestations, establishes a novel framework that integrates genotypes, environments, and phenotypes under a unified, dynamic, and complex network setting.

The theories and ontologies can interact together as semantic frameworks to support integrative vaccinology research. The theories provide the basic structure, the contents and logics of the entities for semantic ontological representation. Meanwhile, without ontology, it is difficult to represent multi-layer, condition-dependent complex network systems covered by the theories. Currently we are developing the Interaction Network Ontology (INO) [13] and Human INO (HINO) [25]. These ontologies will be combined with VO/OAE/OVAE to further support vaccine-specific interaction network representation and analyses. The integration between the ontologies and theories, together with various systems biology approaches, will ensure better integrative representations and analyses of the basic molecular interactions and clinical phenotypes associated with vaccine-induced intended protective/ therapeutic immune responses and unintended adverse events.

\section{Acknowledgements}

The development of VO, OAE, and OVAE was supported by an NIH NIAID grant 1R01AI081062. The development of VICO was supported by a MCubed fund at the University of Michigan.

\section{References}

1. Simon WL, Salk HM, Ovsyannikova IG, Kennedy RB, Poland GA (2014) Cytokine production associated with smallpox vaccine responses. Immunotherapy 6: 1097-1112. [Crossref]

2. Burns CC, Diop OM, Sutter RW, Kew OM (2014) Vaccine-derived polioviruses. $J$ Infect Dis 210 Suppl 1: S283-293. [Crossref]

3. He Y, Cowell L, Diehl AD, Mobley H, Peters B, et al. (2009) VO: Vaccine Ontology The 1st International Conference on Biomedical Ontology (ICBO-2009). Buffalo, NY, USA: Nature Precedings: http://precedings.nature.com/documents/3552/version/1.

4. Ozgür A, Xiang Z, Radev DR, He Y (2011) Mining of vaccine-associated IFN- $\gamma$ gene interaction networks using the Vaccine Ontology. J Biomed Semantics 2 Suppl 2: S8. [Crossref]

5. Xiang Z, Todd T, Ku KP, Kovacic BL, Larson CB, et al. (2008) VIOLIN: vaccine investigation and online information network. Nucleic Acids Res 36: D923-928. [Crossref]

6. He Y, Racz R, Sayers S, Lin Y, Todd T, et al. (2014) Updates on the web-based VIOLIN vaccine database and analysis system. Nucleic Acids Res 42: D1124-1132. [Crossref]

7. Yang B, Sayers S, Xiang Z, He Y (2011) Protegen: a web-based protective antigen database and analysis system. Nucleic Acids Res 39: D1073-1078. [Crossref]

8. Sayers S, Ulysse G, Xiang Z, He Y (2012) Vaxjo: a web-based vaccine adjuvan database and its application for analysis of vaccine adjuvants and their uses in vaccine development. J Biomed Biotechnol 2012: 831486. [Crossref]

9. Racz R, Chung M, Xiang Z, He Y (2013) Systematic annotation and analysis of "virmugens" - virulence factors whose mutants can be used as live attenuated vaccines. Vaccine 31: 797-805. [Crossref]

10. He Y, Racz R, Sayers S, Lin Y, Todd T, et al. (2014) Updates on the web-based VIOLIN vaccine database and analysis system. Nucleic Acids Res 42: D1124-1132. [Crossref]

11. Deng S, Martin C, Patil R, Zhu F, Zhao B, et al. (2015) Vaxvec: The first web-based recombinant vaccine vector database and its data analysis. Vaccine 33: 6938-6946. [Crossref]

12. Hur J, Xiang Z, Feldman EL, He Y (2011) Ontology-based Brucella vaccine literature indexing and systematic analysis of gene-vaccine association network. BMC Immunol 12: 49. [Crossref]

13. Hur J, Özgür A, Xiang Z, He Y (2015) Development and application of an interaction network ontology for literature mining of vaccine-associated gene-gene interactions. $J$ Biomed Semantics 6: 2. [Crossref]

14. Marcos E, Zhao B, He Y (2013) The Ontology of Vaccine Adverse Events (OVAE) and its usage in representing and analyzing adverse events associated with US-licensed human vaccines. J Biomed Semantics 4: 40. [Crossref]

15. He Y, Sarntivijai S, Lin Y, Xiang Z, Guo A, et al. (2014) OAE: The Ontology of Adverse Events. J Biomed Semantics 5: 29. [Crossref]

16. Lin Y, Zheng H and He Y (2016) VICO: Ontology-based representation and integrative analysis of vaccination informed consent forms. J Biomed Semantics 7: 20

17. Sarntivijai S, Zhang S, Jagannathan DG, Zaman S, Burkhart KK, et al. (2016) Linking MedDRA ${ }^{\circledR}$-Coded Clinical Phenotypes to Biological Mechanisms by the Ontology of Adverse Events: A Pilot Study on Tyrosine Kinase Inhibitors. Drug Saf. [Crossref]

18. Ashburner M, Ball CA, Blake JA, Botstein D, Butler H, et al. (2000) Gene ontology: tool for the unification of biology. The Gene Ontology Consortium. Nat Genet 25: 25-29. [Crossref]

19. Poland GA, Ovsyannikova IG, Jacobson RM, Smith DI (2007) Heterogeneity in vaccine immune response: the role of immunogenetics and the emerging field of vaccinomics. Clin Pharmacol Ther 82: 653-664. [Crossref]

20. Poland GA, Ovsyannikova IG, Jacobson RM (2009) Application of pharmacogenomics to vaccines. Pharmacogenomics 10: 837-852. [Crossref]

21. Poland GA, Ovsyannikova IG, Kennedy RB, Haralambieva IH, Jacobson RM (2011) Vaccinomics and a new paradigm for the development of preventive vaccines against viral infections. OMICS 15: 625-636. [Crossref] 
He Y (2016) Integrative representations and analyses of vaccine-induced intended protective immunity and unintended adverse events using ontology-based and theory-guided approaches

22. Poland GA, Kennedy RB, McKinney BA, Ovsyannikova IG, Lambert ND, et al. (2013) Vaccinomics, adversomics, and the immune response network theory: individualized vaccinology in the 21st century. Semin Immunol 25: 89-103. [Crossref]

23. He Y (2014) Ontology-supported research on vaccine efficacy, safety and integrative biological networks. Expert Rev Vaccines 13: 825-841. [Crossref]
24. He Y (2016) Ontology-based vaccine and drug adverse event representation and theoryguided systematic causal network analysis toward integrative pharmacovigilance research. Current Pharmacology Reports 2: 113-128.

25. He Y and Xiang Z. (2013) HINO: a BFO-aligned ontology representing human molecular interactions and pathways. arXiv: arXiv:1311.3355.

Copyright: $@ 2016 \mathrm{He}$ Y. This is an open-access article distributed under the terms of the Creative Commons Attribution License, which permits unrestricted use, distribution, and reproduction in any medium, provided the original author and source are credited. 